\title{
Comparison of two techniques for toric intraocular lens implantation: hydroimplantation versus ophthalmic viscosurgical devices
}

\author{
Yueqin Chen, Qian Cao, Chunyan Xue* and Zhenping Huang*
}

\begin{abstract}
Background: To compare the results between hydroimplantation of a single-piece, acrylic foldable toric intraocular lens (IOLs) and conventional implantation using an ophthalmic viscosurgical device (OVD).

Methods: In this study, 60 eyes with cataract and preexisting regular corneal astigmatism of 1.0 to 3.0 diopters (D) underwent the implantation of the AcrySof toric IOLs (Alcon Laboratories, Inc.). The patients were randomly assigned to a conventional implantation technique with an OVD or a hydroimplantation technique. Comparison of preoperative and postoperative parameters was performed using paired Student $t$ tests, and independent Student $t$ test was used to compare between the two groups.

Results: Three months postoperatively, the mean subjective astigmatism was $0.45 \mathrm{D} \pm 0.24$ (SD) in the OVD group and $0.49 \pm 0.29 \mathrm{D}$ in the hydroimplantation group $(P=0.492)$. The mean endothelial cell density (ECD) loss was $7.54 \% \pm 0$. $82 \%$ and $7.32 \% \pm 0.59 \%$, respectively $(P=0.117)$. The mean absolute IOL rotation was $4.77 \pm 2.32$ degrees and $4.70 \pm 1$. 95 degrees, respectively $(P=0.334)$. The mean time for $\mathrm{IOL}$ implantation was $71.50 \pm 8.10 \mathrm{~s}$ and $37.60 \pm 3.90 \mathrm{~s}$, respectively $(P<0.001)$. Two hours, 1 day, 1 week, 1 month, and 3 months postoperatively, there was no significant difference in IOP between the two groups $(P>0.05)$, although IOP two hours postoperatively seemed to be a little higher in the OVD group.

Conclusions: Compared with the use of OVDs for toric IOLs implantation, the hydroimplantation technique provided advantages of increased efficiency, reduced surgical time and cost, and no concerns of OVD-induced elevated IOP.
\end{abstract}

Trial registration: Current Controlled Trials ISRCTN55696872, Retrospectively registered (Date of registration: 25 March 2018).

Keywords: Endothelial cell density, Hydroimplantation, Intraocular pressure, Ophthalmic viscosurgical device, Toric intraocular lens

\footnotetext{
* Correspondence: xuechunyancn@163.com; hzpjlyy@hotmail.com

Department of Ophthalmology, Jinling Hospital, School of Medicine, Nanjing

University, 305 East Zhongshan Road, Nanjing, People's Republic of China
}

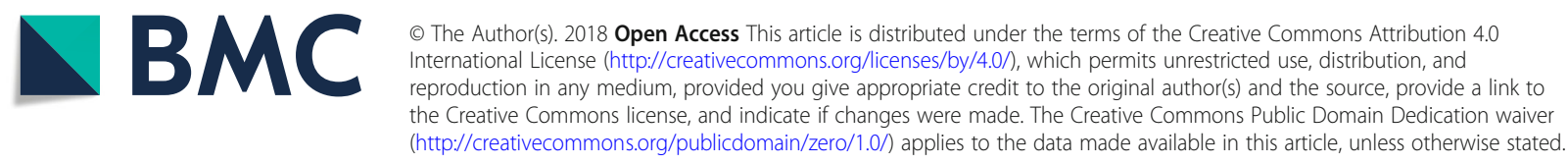




\section{Background}

It is estimated that $25 \%$ to $40 \%$ of cataract patients have astigmatism more than 1.0 diopters (D) $[1,2]$. With the increasing demands for good refractive outcomes after cataract surgery, toric intraocular lenses (IOLs) have been widely used to correct preoperative corneal astigmatism during surgery. Many studies have shown that the toric IOL implantation offers a predictable and stable procedure for the correction of preexisting corneal astigmatism, and induces a low amount of higher-order aberration $[1,3-6]$. The key to achieve a good reduction of astigmatism is the precise alignment of the toric IOL axis on the planned meridian. In the standard procedure, after the injection of the toric IOL, the ophthalmic viscosurgical device (OVD) should be aspirated from the anterior chamber, including from behind the lens. This procedure is crucial for the toric IOL axis being finally positioned on the planned meridian. And it could be especially difficult when one haptic of the toric IOL is located adjacent to the main incision.

Hydroimplantation,implantation of a foldable IOL without an OVD, was introduced by Tak [7], and adopted and modified by other surgeons later [8-12]. To our knowledge, there has not been any study about comparison between hydroimplantation and conventional technique for the implantation of toric IOLs. However, the hydroimplantation technique can make the toric IOL implantation much easier by skipping two steps of rotation, and the toric IOL could be rotated to its final meridian directly.

The aim of this study was to evaluate and compare the clinical results between the OVD group and the hydroimplantation group for the implantation of a single-piece, acrylic foldable toric IOL.

\section{Methods}

\section{Patient population}

This randomized prospective study comprised 60 eyes with cataract and preexisting regular corneal astigmatism between 1.0 and 3.0 diopters (D) who had implantation of an AcrySof toric IOL (Alcon Laboratories, Inc.). The surgeries were performed between Jan 2012 and May 2014 by one experienced surgeon (Dr. Chunyan Xue) at the Refractive Center, Department of Ophthalmology, Jinling Hospital, Nanjing, China. The eyes were randomized prospectively into two groups (the OVD group and the hydroimplantation group) according to the statistical random table. The study protocol was approved by the Ethical Committee of Jinling Hospital. All patients were fully informed of the details and possible risks of the procedure, and written informed consents were obtained from all the patients. Described research adhered to the tenets of the Declaration of Helsinki.

Inclusion criteria were age-related cataract with preoperative corneal astigmatism between 1.0 and $3.0 \mathrm{D}$ and nucleus sclerosis up to grade 3. Preoperative corneal astigmatism was measured by keratometry (IOLMaster, Zeiss Humphrey, Carl Zeiss Meditec, Inc., Dublin, CA 94568). Exclusion criteria were history of previous ocular surgery, pupil size less than $7.5 \mathrm{~mm}$ after dilatation, anterior chamber less than $2.25 \mathrm{~mm}$, compromised endothelial cell function, corneal disorder, complicated cataract, glaucoma, pseudoexfoliation, severe myopia, and diabetic retinopathy.

\section{Preoperative assessment}

Preoperative evaluations included subjective refraction, uncorrected visual acuity (UCVA), best corrected visual acuity (BCVA) (Snellen or "E" chart), keratometry (IOLMaster, Zeiss Humphrey, Carl Zeiss Meditec, Inc., Dublin, CA 94568), corneal topography (Humphrey ATLAS Corneal Topography System, Carl Zeiss Meditec, Inc., Dublin, CA 94568), intraocular pressure (IOP) (Computerized Tonometer CT-80A, Topcon Corp. Tokyo, Japan), endothelial cell density (ECD) (SP-3000P, Topcon Corp. Tokyo, Japan), slitlamp examination (PS-11E, Topcon Corp. Tokyo, Japan), and indirect fundus examination.

\section{Intraocular Lens}

The AcrySof toric IOL SN6ATT (Alcon Laboratories, Inc., Fort Worth, TX, USA) has open-loop modified Lhaptics with 3 reference dots on each side that mark the axis of the cylinder on its posterior surface. The IOL can filter ultraviolet and blue light and is a single-piece IOL made of hydrophobic acrylic material with a refractive index of 1.55 , a $6.0-\mathrm{mm}$ optic diameter, and a $13.0-\mathrm{mm}$ overall length. The spherical IOL power was calculated using the IOLMaster (Carl Zeiss Meditec AG) and the SRK/T formula. The cylinder power and the alignment axis were calculated using the Acrysof toric calculator available from Alcon Laboratories (www.acrysoftoriccal culator.com). The main incision was created at $135^{\circ}$ and the surgical induced astigmatism was set as $0.5 \mathrm{D}$ at $45^{\circ}$ according to the data of previous operations of the same surgeon.

\section{Surgical technique}

Preoperatively, limbal reference marks of the astigmatic axis were made under a slit lamp directly [13]. The patients were sitting at the slit lamp and instructed to look at a distant target at head height with the fellow eye. The slit light was centered on the apex of the cornea and turned in the steep astigmatic meridian in the orthograde position using the rotator switch of the slit light. Then, two points of the astigmatic meridian at the limbus were marked with a marking pen. All of the markings were done by the surgeon.

$5 \%$ Tropicamide with 5\% phenylephrine eye drops were used for preoperative pupillary dilation. The surgery 
was performed using the Infiniti Vision System (Alcon Laboratories, Inc.). In both groups, after topical anesthesia with oxybuprocaine hydrochloride $0.4 \%$ eye drops, a 2 step superior clear corneal incision with an approximate $2.0 \mathrm{~mm}$ chord length was created with a $2.2 \mathrm{~mm}$ Intrepid dual-bevel slit knife (Alcon Laboratories, Inc.), and a side port was created at 2 o'clock at the limbus using a paracentesis knife (Alcon Laboratories, Inc.). After the anterior chamber was filled with DiscoVisc OVD (Alcon Laboratories, Inc.), a continuous curvilinear capsulorhexis measuring 5.0 to $5.5 \mathrm{~mm}$ in diameter was generated using capsular forceps. Hydrodissection and hydrodelineation were performed. Following in-the-bag phacoemulsification using the quick-chop technique, the rest of the cortex was removed by irrigation/aspiration using the Intrepid silicone-sleeved coaxial system (Alcon Laboratories, Inc.).

In the OVD group, the anterior chamber and the capsular bag was filled with DiscoVisc (Alcon Laboratories, Inc.). The toric IOL was placed in the cartridge which was lubricated with DiscoVisc. The IOL was then implanted into the capsular bag using a D cartridge mounted on a Monarch II injector (Alcon Laboratories, Inc.) by the surgeon. The injector was held with the left hand and rotated for advancement of the IOL with the other hand. After the IOL was injected into the capsular bag, gross alignment was achieved by rotating the IOL clockwise until it was placed 20-30 degrees short of the planned axis. After the DiscoVisc was thoroughly removed from the eye and from behind the IOL, the IOL was rotated to its final position by exactly aligning the reference marks on the toric IOL with the alignment axis marks. A final check was made after the anterior chamber was filled and the incisions were hydrated.

In the hydroimplantation group, following the removal of any residual cortical material, balanced salt solution (BSS) (Alcon Laboratories, Inc.) was used to maintain the anterior chamber shape instead of the DiscoVisc. To implant the IOL, the irrigation cannula was inserted into the anterior chamber through the left side port. The IOL was gradually injected into the eye through the tip of the cartridge with the assistance of an assistant who screwed the end of the injector, and the tip of the irrigation cannula could be used to guide the IOL into the capsular bag. With the help of the irrigation cannula tip and the small tip of a cyclodialysis spatula, the IOL was rotated to its final position by exact alignment of the reference marks on the toric IOL with the alignment axis marks. Finally, the incisions were hydrated (Fig. 1).

Tobramycin-dexamethasone ointment was applied to the eye at the end of the surgery. Postoperatively, tobramycin and dexamethasone eye drops were applied topically every hour. The eye drops were tapered after 1 week and then discontinued after 2 weeks.

\section{Postoperative assessment}

Postoperative IOP were performed at $2 \mathrm{~h}, 1$ day, 1 week, 1 month, and 3 months after surgery. Postoperative UDVA, ECD, refractive astigmatism (keratometry: IOLMaster, Zeiss Humphrey, Carl Zeiss Meditec, Inc., Dublin, CA 94568), IOL rotation were also evaluated after surgery. The time for the IOL implantation was recorded during the surgery. Rotation of the toric IOL was evaluated by a slit lamp: A thin slit beam was rotated to the axis markings of the IOL, and then the orientation of the IOL was estimated in 1-degree steps [14]. Astigmatism vector analysis was performed using Thibos and Horner's power vector notation [15].

\section{Statistical analysis}

The results were analyzed using SPSS software (version 17.0, SPSS, Inc.). Data were presented as Mean \pm SD. Visual acuity was converted to logarithm of the minimum angle of resolution $(\log M A R)$ before statistical analysis. For quantitative variables, independent Student $t$ test was used to compare between the two groups. Comparison of preoperative and postoperative parameters was performed using paired Student $t$ tests. A normal distribution check (Kolmogorov-Smirnov test) was performed to validate the use of a Student $t$ test. Qualitative variables were analyzed using the Chi-square test. A $P$ value less than 0 . 05 was considered statistically significant.

\section{Results}

Sixty eyes were included in this study. All the eyes had successful operations with total surgical time less than $20 \mathrm{~min}$, and no eye had posterior capsule rupture or other complications during the surgery. Table 1 shows the demographic data. No statistically significant differences in age, sex, UCVA, BCVA, IOP, ECD, or refractive astigmatism were observed between the two groups before surgery $(P>0.05)$.

\section{Visual acuity}

After surgery, UCVA improved significantly in all the patients $(P<0.001)$. Three months postoperatively, the mean UCVA was $0.19 \pm 0.11 \log$ MAR (range 0.00 to 0 . $40 \log M A R)$ in the OVD group and $0.19 \pm 0.12 \log$ MAR (range 0.00 to $0.40 \log \mathrm{MAR}$ ) in the hydroimplantation group. There was no statistically significant difference in UCVA between the two groups $(P=0.550)$.

\section{Refractive outcomes}

There was a significant reduction of refractive astigmatism in the two groups after surgery $(P<0.001)$. Three months postoperatively, the mean refractive astigmatism was $0.45 \pm 0.24 \mathrm{D}$ (range 0.14 to $1.01 \mathrm{D}$ ) in the OVD group and $0.49 \pm 0.29 \mathrm{D}$ (range 0.12 to $1.28 \mathrm{D}$ ) in the hydroimplantation group. There was no statistically significant difference in refractive astigmatism between 


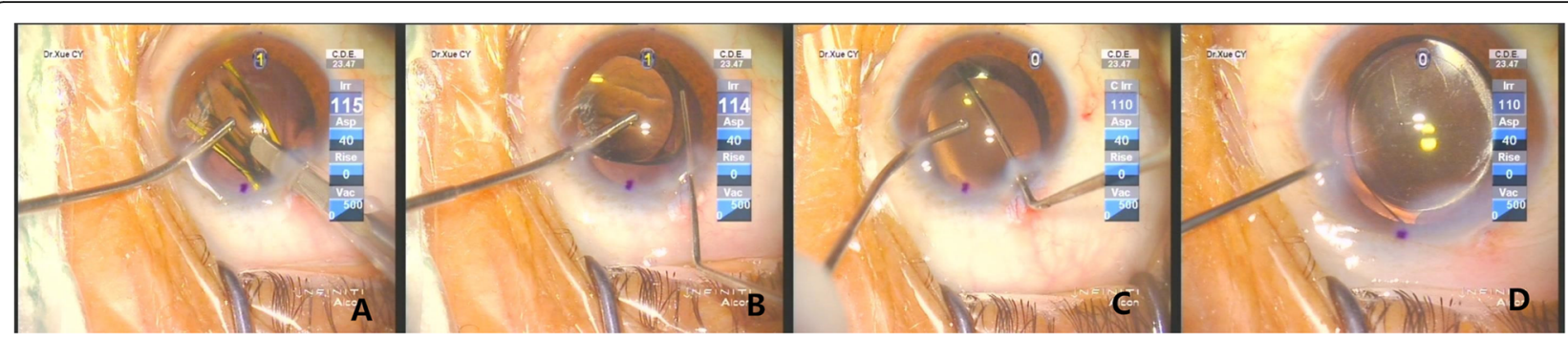

Fig. 1 Implantation of a toric IOL. a The leading haptic is inserted into the capsular bag with the help of the irrigation cannula tip. $\mathbf{b}$ The trailing haptic is dialed and inserted into the capsular bag with the help of the small tip of a cyclodialysis spatula. c The toric IOL is rotated to its planned position directly. $\mathbf{d}$ A final check that the axis of the IOL is on the planned meridian and the incisions are hydrated

the two groups $(P=0.492)$. Figures 2 and 3 show the linear regression about the expected vs achieved spherical equivalent (SE) 3 months postoperatively in the 2 groups, and 28 eyes (93.3\%) in the OVD group and 27 eyes $(90.0 \%)$ in the hydroimplantation group were within $\pm 1.50 \mathrm{D}$ of target refraction.

Figure 4 shows the postoperative astigmatism power vector in both groups. Three months postoperatively, the mean Jackson cross-cylinder at J0 and J45 was $0.08 \pm 0.19$ $\mathrm{D}$ (range -0.26 to $0.43 \mathrm{D}$ ) and $0.08 \pm 0.14 \mathrm{D}$ (range -0.20 to $0.41 \mathrm{D})$ respectively in the OVD group, and $0.00 \pm 0.23$ $\mathrm{D}$ (range -0.62 to $0.46 \mathrm{D}$ ) and $0.09 \pm 0.15 \mathrm{D}$ (range -0.25 to $0.49 \mathrm{D})$ respectively in the hydroimplantation group. There was no statistically significant difference in $\mathrm{J0}(P=$ $0.287)$ or $J 45(P=0.992)$ between the two groups.

\section{Endothelial cell density}

Three months after surgery, the mean ECD was $2235.50 \pm$ 294.33 cells $/ \mathrm{mm}^{2}$ (range 1723.4 to 2765.8 cells $/ \mathrm{mm}^{2}$ ) in the OVD group and $2343.59 \pm 287.43$ cells $/ \mathrm{mm}^{2}$ (range 1712.6

Table 1 Demographic data at baseline

\begin{tabular}{llll}
\hline Variables & OVD group & $\begin{array}{l}\text { Hydroimplantation } \\
\text { group }\end{array}$ & $P$ value \\
\hline Number & 30 & 30 & \\
Gender (M/F) & $10 / 15$ & $11 / 17$ & 0.480 \\
Age (years) & $69.53 \pm 10.33$ & $67.53 \pm 9.14$ & 0.513 \\
UCVA (logMAR) & $0.93 \pm 0.25$ & $0.81 \pm 0.14$ & 0.536 \\
BCVA (logMAR) & $0.67 \pm 0.24$ & $0.68 \pm 0.22$ & 0.483 \\
ECD (cells/mm²) & $2418.66 \pm 322.97$ & $2529.71 \pm 317.50$ & 0.939 \\
IOP (mmHg) & $16.15 \pm 2.48$ & $15.29 \pm 2.31$ & 0.803 \\
Refractive astigmatism (D) & $2.29 \pm 0.44$ & $2.10 \pm 0.42$ & 0.804 \\
Toric IOL model, n (\%) & & & \\
T3 & $9(30.0)$ & $11(36.7)$ & \\
T4 & $9(30.0)$ & $8(26.7)$ & \\
T5 & $11(36.7)$ & $10(33.3)$ & \\
T6 & $1(3.3)$ & $1(3.3)$ & \\
\hline
\end{tabular}

OVD ophthalmic viscosurgical device, UCVA uncorrected visual acuity, BCVA best-corrected visual acuity, ECD endothelial cell density, IOP intraocular pressure, $D$ diopters to 2699.4 cells $/ \mathrm{mm}^{2}$ ) in the hydroimplantation group. The mean ECD loss was $7.54 \% \pm 0.82 \%$ and $7.32 \% \pm 0.59 \%$, respectively $(P=0.117)$.

\section{Intraocular pressure}

Table 2 shows the IOP in $2 \mathrm{~h}, 1$ week, 1 month, and 3 months after surgery. There was no significant difference in IOP between the two groups during the follow up $(P>0.05)$, although IOP two hours postoperatively seemed to be a little higher in the OVD group.

\section{Intraocular lens rotation}

Three months postoperatively, the mean absolute IOL rotation relative to the intended meridian was $4.77 \pm 2$. 32 degrees (range 1 to 10 degrees) in the OVD group and $4.70 \pm 1.95$ degrees (range 2 to 10 degrees) in the hydroimplantation group. There was no statistically significant difference in IOL rotation between the two groups $(P=0.334)$ (Fig. 5).

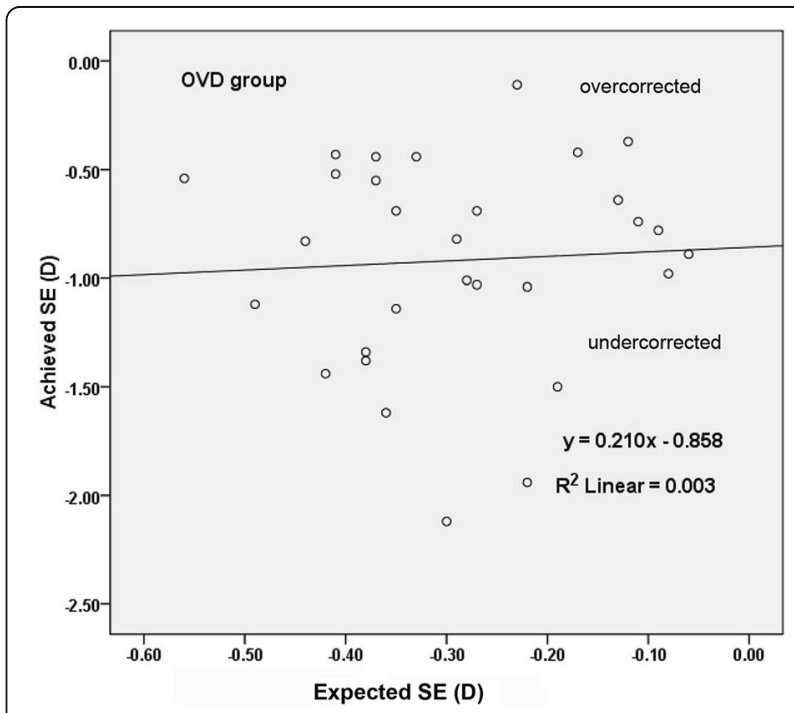

Fig. 2 Achieved SE correction versus expected SE correction 3 months postoperatively in OVD group. (SE spherical equivalent, OVD ophthalmic viscosurgical device) 


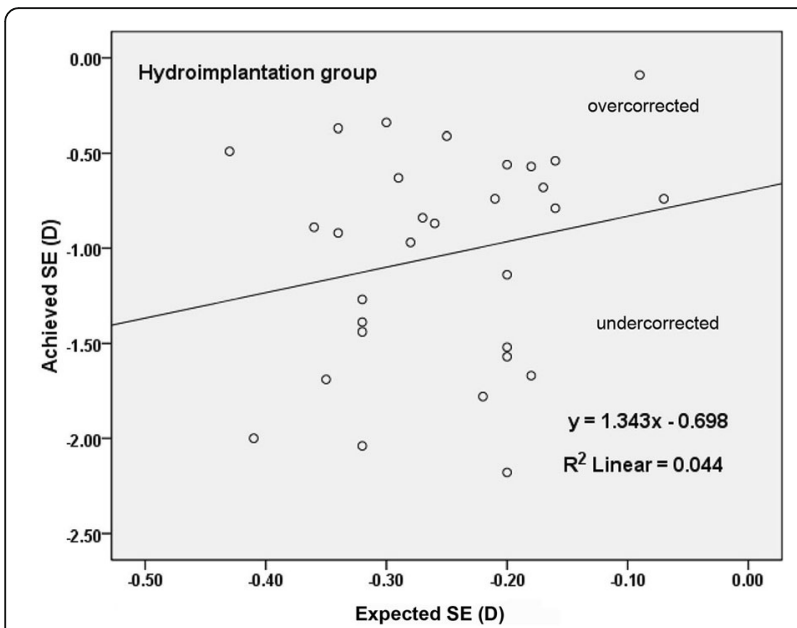

Fig. 3 Achieved SE correction versus expected SE correction 3 months postoperatively in hydroimplantation group. (SE spherical equivalent)

\section{Time for intraocular lens implantation}

The time for the IOL implantation was calculated from the end of complete removal of the cortex to the beginning of incision hydrated. The mean time for the toric IOL implantation was $71.50 \pm 8.10 \mathrm{~s}$ in the OVD group and $37.60 \pm 3.90 \mathrm{~s}$ in the hydroimplantation group. The time for the IOL implantation was much less in the hydroimplantation group $(P<0.001)$.

\section{Discussion}

There are many studies about foldable IOLs implantation using anterior chamber infusion, but no comparison study

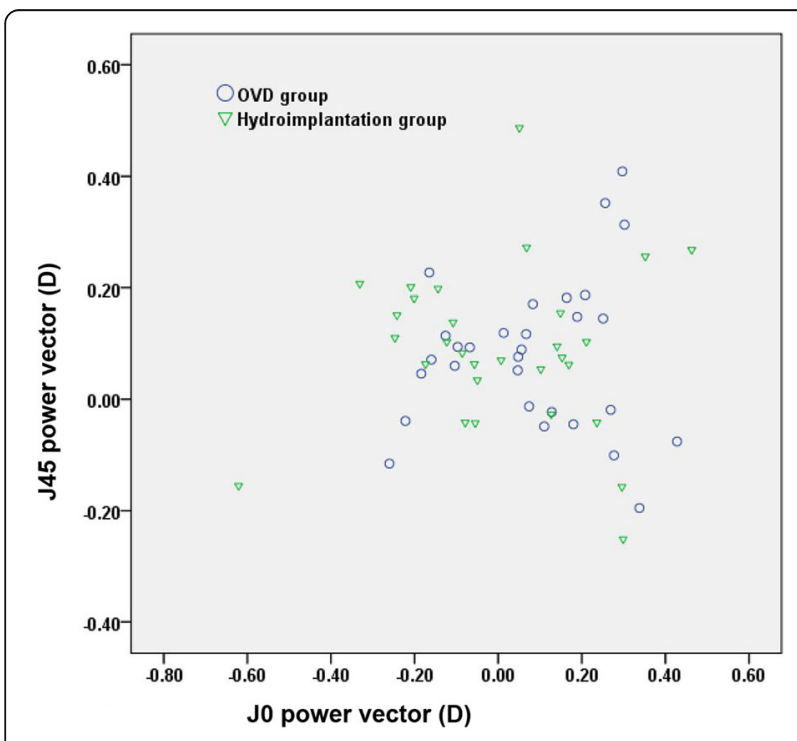

Fig. 4 Astigmatism vectors. The $x$-axes represent the J0 power vector (D). The $y$-axes represent the J45 power vector (D). (OVD ophthalmic viscosurgical device, 10 Jackson cross-cylinder, axes at 0 degree and 90 degree, 145 Jackson cross-cylinder, axes at 45 degree and 135 degree)
Table 2 Mean IOP change $(\mathrm{mmHg})$ postoperatively

\begin{tabular}{llll}
\hline Time & OVD group & Hydroimplantation group & $P$ value \\
\hline $2 \mathrm{~h}$ & $16.25 \pm 3.38$ & $14.79 \pm 3.02$ & 0.830 \\
1 day & $14.98 \pm 3.52$ & $14.13 \pm 2.83$ & 0.119 \\
1 week & $13.45 \pm 2.56$ & $13.56 \pm 2.35$ & 0.609 \\
1 month & $14.15 \pm 1.72$ & $13.47 \pm 1.78$ & 0.715 \\
3 months & $14.70 \pm 1.99$ & $14.08 \pm 1.93$ & 0.981 \\
\hline IOP intraocular pressure, OVD ophthalmic viscosurgical device &
\end{tabular}

about toric IOLs implantation. In this study, we find that the advantages of the hydroimplantation over OVDs are increased efficiency and reduced cost [16]. During the IOL implantation, the IOL can be rotated to its final position directly, skipping two steps of rotation using hydroimplantation compared with OVDs. Therefore, the hydroimplantation technique leads to deceased surgery time and increased efficiency, which is important in high-volume surgical centers. In our study, the mean time for the toric IOL implantation was $71.50 \mathrm{~s}$ in the OVD group and 37. $60 \mathrm{~s}$ in the hydroimplantation group. The time for the IOL implantation was much less in the hydroimplantation group. Additionally, if smaller sized OVD syringes become available in the future, the hydroimplantation technique may reduce costs. And the manufacturer may produce OVD with smaller size and less cost.

UCVA and refractive astigmatism are important parameters of success for cataract patients with preoperative corneal astigmatism undergone implantation of toric IOLs. Three months after surgery, UDVA and refractive astigmatism were improved in both groups, and there were no significant differences between the two groups in UDVA or in refractive astigmatism. Three months postoperatively, the mean absolute IOL rotation relative to the intended meridian was 4.77 degrees with a range from 1 to 10 degrees in the OVD group and 4.70 degrees with a

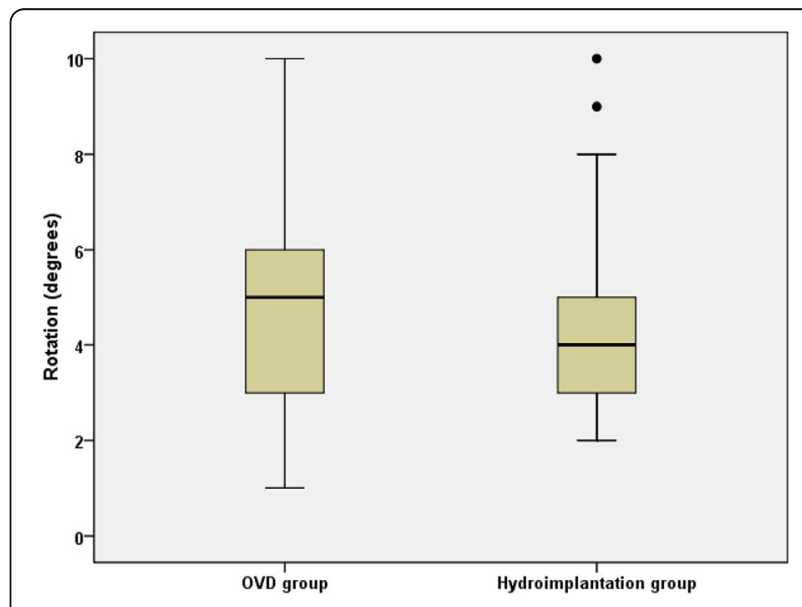

Fig. 5 Absolute axis rotation between the two groups three months postoperatively. (OVD = ophthalmic viscosurgical device) 
range from 2 to 10 degrees in the hydroimplantation group. The rotation of the IOL was similar between the two groups. Complete IOL fixation on the posterior capsule is another advantage of hydroimplantation [10], which is more important for AcySof toric IOL. The stability of AcySof toric IOL in our study is inferior to the findings by Waltz, Hirnschall, and Lee et al. with the mean rotation from 2.2 to 4.1 degrees [17-20]. This result may be related to the evaluation method, preoperative marking, axis placement during surgery, and complete removal of the OVD or using hydroimplantation method.

In this study, BSS was used with a modification of Michael Blumenthal's AC chamber maintainer to maintain the anterior chamber and expand the bag [21]. Nonetheless, this study was carried out on normal corneas and foldable acrylic IOLs. Besides, the technique has limitations in compromised anterior chamber stability compared with OVDs. Therefore, we must be careful to abandon OVDs only when we can safely expand the bag, and it should be evaluated whether it could be applicable for diseased corneas and other IOLs. We do not recommend this technique for novice ophthalmologists. Mackool suggests using this technique in normal cases and to keep the OVD implantation technique for IOLs that open abruptly and complicated cases such as posterior capsular rupture, torn anterior capsulorhexis, and floppy-iris syndrome [9].

In the hydroimplantation method, the irrigation cannula was inserted into the anterior chamber through the left side port with the surgeon's left hand and the injector held with the other hand. It was not a 2-handed technique when the IOL was implanted using a manual Monarch injector. The IOL was injected into the eye with the aid of an assistant who screwed the end of the injector. If a motorized IOL injector was used, the injection procedure with hydroimplantation would be a 2-handed technique [22]. Furthermore, this procedure would be easy and safe when the eye was stabilized by the irrigation cannula in the side port.

As Lee reported that using BSS for maintaining the anterior chamber without OVD during IOL implantation did not cause a significant difference in ECD loss 3 months after surgery, our study showed the same results [11]. There was no significant difference in ECD loss between the two groups in our study. Lee revealed that use of BSS during IOL implantation resulted in reduction in postoperative IOP spike compared with the use of OVD [11]. But in our study, we did not find any difference in postoperative IOP between the two groups, although IOP two hours postoperatively seemed to be a little higher in the OVD group (16.25 mmHg in the OVD group versus 14 . $79 \mathrm{mmHg}$ in the hydroimplantation group). This may be due to the small sample in our study or incomplete removal of the OVD in Lee's study. Of note, previously vitrectomized eyes were included in Lee's study which were absent in our study. The study by Studeny et al. found no influence of the implantation technique (OVD or hydroimplantation) on postoperative IOP changes, which was comparable to our study [9]. However, larger studies may be needed to evaluate the influence of the two techniques on postoperative IOP, especially at different time point on the first postoperative day.

\section{Conclusion}

In conclusion, the hydroimplantation technique provides comparable outcomes to the conventional technique using OVDs for the implantation of toric IOLs, however, it has the advantages of increased efficiency, reduced surgical time and cost (if smaller size of OVD is available), and no concerns of OVD-induced elevated IOP. Furthermore, this technique is useful for the alignment of toric IOLs during the surgery, and the stability of the toric IOLs after surgery. However, the hydroimplantation cannot completely replace the OVD technique since this technique has advantages in complex cases. Importantly, this technique is only recommended to experienced surgeon, for novice ophthalmologists may cause some tough intraoperative complications. Thus, hydroimplantation technique may be an alternative method for the implantation of a single-piece, acrylic foldable toric IOL in some cases.

\section{Abbreviations}

BBS: Balanced salt solution; BCVA: Best-corrected visual acuity; ECD: Endothelial cell density; IOLs: Intraocular lens; IOP: Intraocular pressure; LogMAR: Logarithm of the minimum angle of resolution; OVD: Ophthalmic viscosurgical device; SE: Spherical equivalent; UCVA: Uncorrected visual acuity

\section{Acknowledgements}

The authors would like to acknowledge all members of the Department of Ophthalmology of Jinling Hospital.

\section{Funding}

The whole study was performed without any funding.

\section{Availability of data and materials}

The datasets used and/or analysed during the current study are available from the corresponding authors on reasonable request.

\section{Authors' contributions \\ YC participated in the analysis of the data and drafting the manuscript. QC retrieved information from medical records and supplied technical support. CX planned the study, performed the surgeries, and retrieved information from medical records. ZH participated in the study design, interpretation of the data, and the revision of the manuscript. All authors read and approved the final manuscript.}

\section{Ethics approval and consent to participate}

The study protocol was approved by the Ethical Committee of Jinling Hospital. All patients were fully informed of the details and possible risks of the procedure, and written informed consents were obtained from all the patients. Described research adhered to the tenets of the Declaration of Helsinki.

\section{Competing interests}

The authors declare that they have no competing interests.

\section{Publisher's Note}

Springer Nature remains neutral with regard to jurisdictional claims in published maps and institutional affiliations. 
Received: 3 November 2016 Accepted: 3 April 2018

Published online: 24 April 2018

\section{References}

1. Leon P, Pastore MR, Zanei A, Umari I, Messai M, Negro C, et al. Correction of low corneal astigmatism in cataract surgery. Int J Ophthalmol. 2015;8:719-24.

2. Bachernegg A, Ruckl T, Strohmaier C, Jell G, Grabner G, Dexl AK. Vector analysis, rotational stability, and visual outcome after implantation of a new aspheric Toric IOL. J Refract Surg. 2015;31:513-20.

3. Bissen-Miyajima H, Negishi K, Hieda O, Kinoshita S. Microincision hydrophobic acrylic aspheric Toric intraocular Lens for astigmatism and cataract correction. J Refract Surg. 2015;31:358-64.

4. Farooqui $\mathrm{JH}$, Koul A, Dutta R, Shroff NM. Management of moderate and severe corneal astigmatism with AcrySof(R) toric intraocular lens implantation - our experience. Saudi J Ophthalmol. 2015;29:264-9.

5. Nagpal R, Sharma N, Vasavada V, Maharana PK, Titiyal JS, Sinha R, et al. Toric intraocular lens versus monofocal intraocular lens implantation and photorefractive keratectomy: a randomized controlled trial. Am J Ophthalmol. 2015;160:479-86. e2

6. Toto L, Vecchiarino L, D'Ugo E, Cardone D, Mastropasqua A, Mastropasqua $R$, et al. Astigmatism correction with toric IOL: analysis of visual performance, position, and wavefront error. J Refract Surg. 2013;29:476-83.

7. Tak H. Hydroimplantation: foldable intraocular lens implantation without an ophthalmic viscosurgical device. J Cataract Refract Surg. 2010;36:377-9.

8. Bohm P. Usefulness of hydroimplantation technique for foldable IOL implantation. J Cataract Refract Surg. 2010;36:1623-4

9. Mackool RJ. Hydroimplantation. J cataract refract Surg. 2010;36:1801. author reply 1801

10. Studeny P, Hyndrak M, Kacerovsky M, Mojzis P, Sivekova D, Kuchynka P. Safety of hydroimplantation: a foldable intraocular lens implantation without the use of an ophthalmic viscosurgical device. Eur J Ophthalmol. 2014;24(6):850.

11. Lee HY, Choy YJ, Park JS. Comparison of OVD and BSS for maintaining the anterior chamber during IOL implantation. Korean J Ophthalmol. 2011;25:15-21.

12. Carifi G, Pitsas C, Zygoura V, Kopsachilis N. Hydroimplantation of intraocular lenses. J Cataract Refract Surg. 2013;39:1281-2.

13. Bayramlar H, Dag Y, Sadigov F. Comments on corneal astigmatic marking methods. J Cataract Refract Surg. 2013;39:966.

14. Zuberbuhler B, Signer T, Gale R, Haefliger E. Rotational stability of the AcrySof SA60TT toric intraocular lenses: a cohort study. BMC Ophthalmol. 2008;8:8.

15. Thibos LN, Horner D. Power vector analysis of the optical outcome of refractive surgery. J Cataract Refract Surg. 2001;27:80-5.

16. Shingleton BJ, Mitrev PV. Anterior chamber maintainer versus viscoelastic material for intraocular lens implantation: case-control study. J Cataract Refract Surg. 2001;27:711-4.

17. Waltz KL, Featherstone K, Tsai L, Trentacost D. Clinical outcomes of TECNIS toric intraocular lens implantation after cataract removal in patients with corneal astigmatism. Ophthalmology. 2015;122:39-47.

18. Hirnschall N, Maedel S, Weber M, Findl O. Rotational stability of a single-piece toric acrylic intraocular lens: a pilot study. Am J Ophthalmol. 2014;157:405-11.

19. Lee JY, Kang KM, Shin JP, Kim IT, Kim SY, Park DH. Two-year results of AcrySof toric intraocular lens implantation in patients with combined microincision vitrectomy surgery and phacoemulsification. Br J Ophthalmol. 2013;97:444-9.

20. Ferreira TB, Almeida A. Comparison of the visual outcomes and OPD-scan results of AMO Tecnis toric and Alcon Acrysof IQ toric intraocular lenses. J Refract Surg. 2012;28:551-5.

21. Grinbaum A, Blumenthal M, Assia E. Comparison of intraocular pressure profiles during cataract surgery by phacoemulsification and extracapsular cataract extraction. Ophthalmic Surg Lasers Imaging. 2003;34:182-6.

22. Khokhar S, Sharma R, Patil B, Aron N, Gupta S. Comparison of new motorized injector vs manual injector for implantation of foldable intraocular lenses on wound integrity: an ASOCT study. Eye (Lond). 2014;28:1174-8.

Ready to submit your research? Choose BMC and benefit from:

- fast, convenient online submission

- thorough peer review by experienced researchers in your field

- rapid publication on acceptance

- support for research data, including large and complex data types

- gold Open Access which fosters wider collaboration and increased citations

- maximum visibility for your research: over $100 \mathrm{M}$ website views per year

At BMC, research is always in progress.

Learn more biomedcentral.com/submissions 